\title{
Preparations for a Plasma Wakefield Acceleration (PWA) experiment at PITZ
}

\author{
M. Gross ${ }^{\mathrm{a}}$, R. Brinkmann ${ }^{\mathrm{b}}$, J. D. Good ${ }^{\mathrm{c}}$, F. Grüner ${ }^{\mathrm{d}, \mathrm{e}}$, M. Khojoyan ${ }^{\mathrm{a}}$, A. Martinez de la Ossa ${ }^{\mathrm{d}}$, J. Osterhoff ${ }^{\mathrm{b}}$, G. Pathak $^{\mathrm{d}}$, C. \\ Schroeder ${ }^{\mathrm{f}}$, F. Stephan ${ }^{\mathrm{a}}$
}

\begin{abstract}
${ }^{a}$ Deutsches Elektronen-Synchrotron (DESY), Platanenallee 6, 15738 Zeuthen, Germany
${ }^{b}$ Deutsches Elektronen-Synchrotron (DESY), Notkestraße 85, 22607 Hamburg, Germany

${ }^{c}$ Karlsruher Institut für Technologie, Kaiserstraße 12, 76131 Karlsruhe, Germany

${ }^{d}$ Universität Hamburg, Luruper Chaussee 149, 22761 Hamburg, Germany

${ }^{e}$ Center for Free-Electron Laser Science, Notkestrasse 85, 22607 Hamburg, Germany

${ }^{f}$ Lawrence Berkeley National Laboratory, 1 Cyclotron Road, Berkeley, CA 94720, USA
\end{abstract}

\begin{abstract}
Self-modulation of particle beams in a plasma was proposed as a new concept to enable plasma wakefield acceleration with long driver beams. An experiment is in preparation at the Photo Injector Test facility at DESY, Zeuthen site (PITZ), to demonstrate and characterize self-modulation of an electron beam. Key elements for this are the highly flexible photocathode laser system and the well-developed beam diagnostics. Preparations for the experiment have started at PITZ. In a first step a suitable insertion point for the plasma cell was determined with beam dynamics simulations. It was decided to use laser ionization to generate the plasma since this technique is capable of providing a homogeneous plasma channel with sufficient size (1 $\mathrm{mm}$ diameter, $60 \mathrm{~mm}$ length) and density $\left(10^{15} \mathrm{~cm}^{-3}\right)$. Two different ways were found to generate the plasma, utilizing either field ionization with a $1 T W$ Ti:Sapphire laser or single photon ionization with a $400 \mathrm{~mJ}$ ArF excimer laser. As opposed to previously realized designs the ionization laser is coupled from the side, orthogonally to the electron beam direction.
\end{abstract}

Keywords:

Plasma wakefield acceleration, self modulation, ionization, plasma cell

\section{Introduction}

Plasma wakefield acceleration (PWFA) was proposed as an ${ }^{26}$ alternative to conventional acceleration methods, promising ac- ${ }_{28}$ celerating fields which are orders of magnitude higher [1]. As ${ }_{29}$ a striking example, energy doubling of $42 \mathrm{GeV}$ electrons has been demonstrated recently in a meter-scale PWFA experiment ${ }_{31}$ [2].

As a follow-up experiment, it has been proposed by the ${ }_{33}^{32}$ AWAKE collaboration to use high energetic proton beams from ${ }_{34}$ CERN to drive a plasma wave which is able to transfer the proton beam energy to accelerate electron beams to the TeV-energy ${ }_{36}$ scale in a single plasma stage [3]. To minimize the needed size ${ }_{37}$ of the plasma stage it is important to have a high acceleration field, which is maximized when the proton bunch rms length ${ }_{39}$ roughly equals the plasma wavelength [3]. For typical plasma densities in the range of $10^{14}$ to $10^{17} \mathrm{~cm}^{-3}$ the bunch length ${ }_{41}$ should therefore be about $100 \mu \mathrm{m}$ to $1 \mathrm{~mm}$. The problem now ${ }_{42}$ is that existing proton machines produce much longer bunches on the order of $10 \mathrm{~cm}$. It was proposed to use plasma-beam in- ${ }_{44}$ stability to modulate the long proton beam at the plasma wave- ${ }_{45}$ length to produce a bunch train which is able to resonantly drive ${ }_{46}$ large plasma waves for acceleration [4]. So far this concept of ${ }_{47}$ self-modulation was only shown analytically and in simulation $([5],[6])$, but not conclusively in experiment (best results so far
5 in [7]). It would be well worth to conduct such self-modulation experiments before attempting the main plasma acceleration experiment to study characteristics as dephasing, hose-instability etc. Since the underlying physics is the same it is valuable to gain insight into the experimental conditions with electron bunches, which are easier to handle, before proceeding to the experiment with proton bunches. It was suggested [8] to utilize the Photo Injector Test facility at DESY, Zeuthen site (PITZ), which offers a unique possibility to study and demonstrate experimentally the self-modulation of long electron bunches in plasma. Therefore it was decided to build a plasma cell and insert it into the PITZ beam line. Favorable circumstances to do this at PITZ are the unique photoinjector laser system which is described in section 2 and the well-developed diagnostics in the PITZ beam line ([9], [10]). This includes as a centerpiece a measurement setup for the longitudinal phase space consisting of a transverse deflecting cavity and a high resolution electron spectrometer which will be commissioned in the near future. Initial simulations showing the feasibility of the PITZ experiment are discussed in section 3. The topics of sections 4 and 5 are beam simulations of the insertion of the plasma cell into the PITZ setup and optical simulations of the ionization of a plasma channel, respectively. The design of the plasma cell is described in section 6 .

Email address: matthias.gross@desy.de (M. Gross) 


\section{Photoinjector laser}

The PITZ photoinjector laser was developed and built by the Max Born Institute Berlin (similar to the system described in [11]). Its purpose is to generate pulse trains to drive the photocathode of the electron linac. A key element of this laser is the pulse shaper [12], which purpose is to generate a temporal flat top pulse from an incoming Gaussian shaped pulse. It contains 13 temperature controlled birefringent crystals mounted on motorized rotation stages. The crystals split the pulses according to their polarization where the relative amplitude of the two output pulses can be varied freely by adjusting the angles of the crystals. The delay between the output pulses is given by the crystal thickness. If adjusted properly the output can be a flat top pulse with rise and fall times given by those of the input Gaussian pulse (about 2 ps for PITZ) and the full width at half maximum (FWHM) length of the pulse can be up to $25 \mathrm{ps}$.
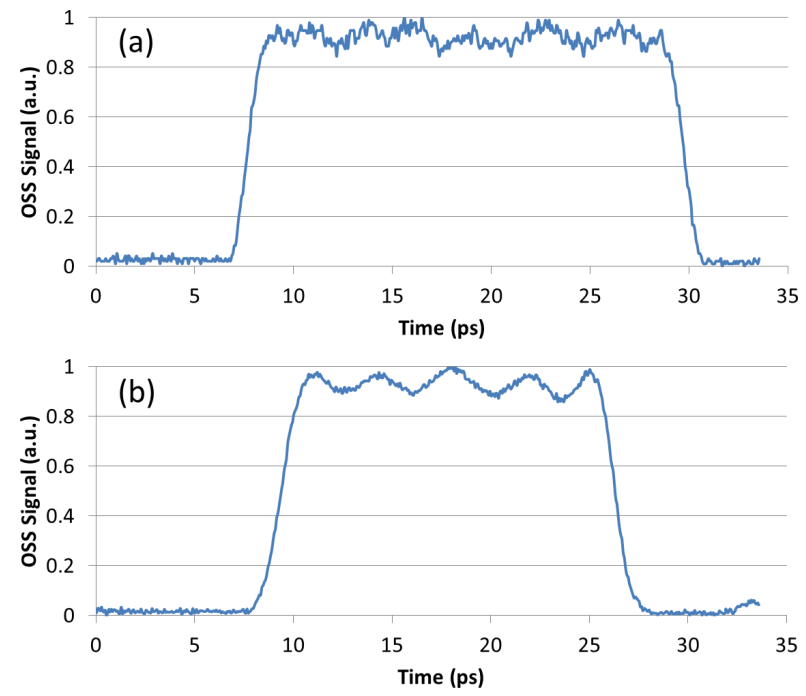

Experimentally measured pulse shapes generated with the PITZ pho- ${ }^{9}$ tocathode laser. Frame (a) shows the nominal flat top which will be used in the100 initial experiments. In frame (b) a shortened flat top pulse with added modula- ${ }_{101}$ tion can be seen.

The nominal flat top pulse which will also be used in the ${ }^{103}$ initial self-modulation experiments is shown in Fig. 1(a). Be- ${ }^{104}$ yond that standard shape the pulse shaper is flexible enough that ${ }^{105}$ other pulse forms can be generated which could be of advan- ${ }^{106}$ tage in plasma acceleration experiments at PITZ. An example ${ }^{107}$ is depicted in Fig. 1(b): here the flat top pulse is shortened, ${ }^{108}$ demonstrating the ability to adjust the pulse length if needed. ${ }^{100}$ Additionally the flat top is modulated which could help to start ${ }^{110}$ the self-modulation process.

\section{PIC Simulation of the PITZ experiment}

In order to evaluate the feasibility of a self-modulation exper-11 iment at PITZ a Particle In Cell (PIC) simulation of the process ${ }_{117}$ was conducted with electron beam parameters typical for PITZ ${ }_{118}$ operation [8], as given in Table 1.
Table 1: PITZ beam parameters used for simulations.

\begin{tabular}{|l|c|}
\hline parameter & value \\
\hline Total charge, $p C$ & 100 \\
Horizontal rms beam size, $\mu \mathrm{m}$ & 42.0 \\
Vertical rms beam size, $\mu \mathrm{m}$ & 42.0 \\
Bunch length in FWHM, $\mathrm{mm}$ & 5.93 \\
Average kinetic energy, $\mathrm{MeV}$ & 21.5 \\
Peak slice current, $A$ & 5.0 \\
Horizontal rms emittance, $\mathrm{mm} \mathrm{mrad}$ & 0.372 \\
Vertical rms emittance, $\mathrm{mm} \mathrm{mrad}_{\text {Peak beam density, } 10^{13} \mathrm{e} / \mathrm{cm}^{3}}^{0.372}$ \\
Pea & 0.9 \\
\hline
\end{tabular}

Shown here is a 3D simulation using the fully relativistic, massively parallel particle-in-cell code OSIRIS [13]. The simulation uses a moving window $(8.4 \mathrm{~mm} \times 3.4 \mathrm{~mm} \times 3.4 \mathrm{~mm})$ that propagates at the speed of light $c$, with resolutions of $k_{p} \Delta z=0.1$ and $k_{p} \Delta y=k_{p} \Delta x=0.04$ ( $k_{p}$ is the wavenumber of the plasma wave) for the longitudinal and transverse sizes, with $2 \times 2 \times 2$ plasma and beam particles per cell. Fig. 2 shows the properties of an electron bunch after $67.6 \mathrm{~mm}$ propagation in a homogeneous plasma with a density of $10^{15} \mathrm{~cm}^{-3}$. At this point, the self-modulation has completely developed and has already reached the saturation point.

Fig. 2(a), shows the electron density distribution (color coded) in the central slice of the simulation (y vs. $\zeta$ at $x=0$ ), clearly indicating the self-modulation: the electron bunch is broken up into several sub-bunches each with the length of about the plasma wavelength which is $1 \mathrm{~mm}$ for the used parameters. The $\mathrm{x}$-axis variable $\zeta=z-v_{\text {beam }} t$, is the co-moving variable with $v_{\text {beam }}$, the velocity of the beam. In Fig. 2(b), the longitudinal electrical field is drawn: negative values indicate acceleration of the electrons along the beam, while positive values means deceleration. As a result, the longitudinal phasespace of the beam (Fig. 2(c)) is modeled according with the wakefields developed by the self-modulation in plasma. The measurement of the longitudinal phase-space of the originally flat-top PITZ beam after the propagation in plasma is the primary goal for the PITZ experiment, since it reflects the main phenomenological features of the self-modulation: the exponential growth in magnitude of the wakefields, leading to a perfectly sinusoidal signature in the longitudinal phase-space, and their dephasing in respect to the beam velocity, which could be estimated by the deviation of the ideal behavior [5]. The expected energy modulation in the experiment is about $400 \mathrm{keV}$ (Fig. 2(c)) with a measured energy spread of the PITZ beam being as low as $60 \mathrm{keV}$, while the simulated energy resolution of the PITZ setup utilizing a transverse deflecting structure (TDS) and a dipole spectrometer (HEDA2) with ideal beam transport is $10 \mathrm{keV}$ [14]. The simulated longitudinal resolution with ideal beam transport is $100 \mu \mathrm{m}$ which is much less than the selfmodulation period of $1 \mathrm{~mm}$ given by the plasma wavelength. Therefore we expect to be able to resolve the signature of the self-modulation in the longitudinal phase space. 

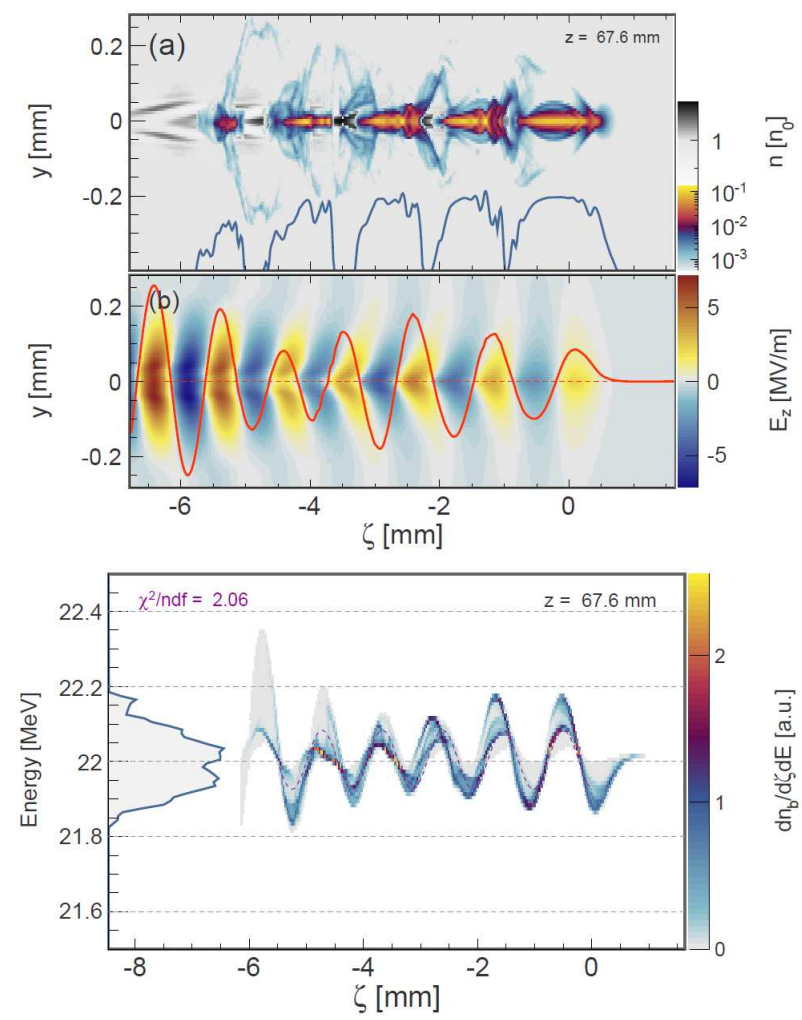

Figure 2: Results of a 3D PIC simulation of self-modulation of the PITZ elec-154 tron beam after $67.6 \mathrm{~mm}$ of propagation in plasma. Frame (a) shows the elec- ${ }_{155}$ tron distribution in the central slice of the simulation (colored palette), and the on-axis values for the beam (blue line). Frame (b) shows the longitudinal elec- ${ }^{156}$ tric field in the slice (colored palette) and on-axis (red line). Frame (c) shows ${ }^{157}$ the longitudinal phase space distribution of the electron beam. The dotted curve ${ }_{158}$ represents a fit to a perfectly sinusoidal signature, strictly valid only in the case ${ }_{159}$ of no de-phasing.

\section{Insertion of the plasma cell into the PITZ setup}

After showing the feasibility of the concept, preparations 164 started to realize the self-modulation experiment in the PITZ 165 beam line (current configuration shown in Fig. 3). First task ${ }^{166}$ here was to find the optimal location to insert the plasma cell,167 which generates the necessary plasma, into the beam line where ${ }^{168}$ high brightness electron beams are generated by the photo effect 169 using a $\mathrm{Cs}_{2} \mathrm{Te}$ cathode and are accelerated in an L-band RF gun 170 cavity in general up to $6.5 \mathrm{MeV}$, corresponding to a momemtum ${ }^{171}$ of about $7 \mathrm{MeV} / \mathrm{c}$ (low energy section). The electron beam gets 172 its final energy of maximal $25 \mathrm{MeV}$ in the high energy section, 173 after the CDS (cut disc structure) booster cavity. The following ${ }^{174}$ dipole is deflecting the beam by $180^{\circ}$ for different studies, par-175 ticularly for the final beam energy measurements. The plasma 176 cell has to be inserted after the booster where nominal electron ${ }_{177}$ beam properties are reached and before the TDS which will ${ }_{178}$ be used to characterize the self-modulated beam. Additionally ${ }_{179}$ it has to be taken into account that several components within ${ }_{180}$ this area cannot be moved since they are needed for other ex-181 periments. This includes the $180^{\circ}$ dipole, the matching section ${ }_{182}$ for the phase space tomography which is located right in front ${ }_{183}$ of the TDS, and several screen stations (H1.S1; 3; 4 and 5 - see $_{184}$
Fig. 4(a)) which are used to measure transverse phase space. This leaves two scenarios allowing a length of the plasma cell plus periphery of about $1 \mathrm{~m}$, which are described and compared in the following.

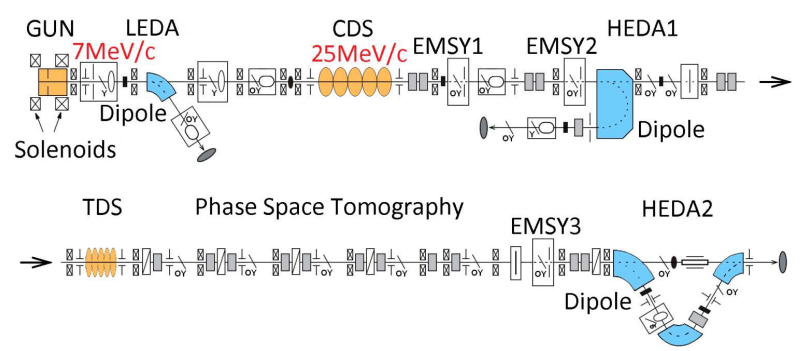

Figure 3: Schematic view of the current PITZ beamline. The electron beam propagates towards the right.

A space charge tracking algorithm (ASTRA) [15] was applied to track the electron beam to the predefined two possible central positions of the plasma chamber (in the middle of the open space in each scenario), i. e. until the point where the beam will enter the plasma. The goal in the simulations was to reach the highest possible electron beam density at the starting point of beam-plasma interaction, by smooth transverse focusing of the beam. In the simulation setup the photocathode laser temporal profile was considered a flat top with $22 \mathrm{ps}$ FWHM length and with $2 \mathrm{ps}$ rise and fall times. The laser rms spot size at the cathode was chosen to be $0.3 \mathrm{~mm}$ for $100 \mathrm{pC}$ bunch charge. The gradient of the RF gun was set to $61 \mathrm{MV} / \mathrm{m}$ corresponding to about $6.7 \mathrm{MeV} / \mathrm{c}$ beam momentum after the gun. The peak field in the second accelerating module (CDS booster) was tuned to achieve a final beam momentum of $21.5 \mathrm{MeV} / \mathrm{c}$. Half a million macroparticles were used in the simulations. In both cases the magnetic strength of the main solenoid was chosen for minimal beam spot size at the first screen station in the high energy section (H1S1). Two pairs of quadrupoles were used to further focus the beam transversely. The simulations were carried out in two steps. Firstly, the electron beam was tracked to $5.1 \mathrm{~m}$ downstream the cathode by using the 2D space charge routine in ASTRA. Quadrupole focusing was applied afterwards for the final beam transverse focusing by using the $3 \mathrm{D}$ space charge settings. The principal part of the simulation setup starting from the first quadrupole in the high energy section until the matching section is illustrated in Fig. 4. The quadrupoles (sketched as diamonds) are introduced in numbers, from one to four, and the screen stations are presented as H1S\#, i. e. H1S1 is the first screen in the high section, etc.

The components which should not be removed / replaced are colored in green. The yellow marked components (H1.S2 and quadrupoles 3 and 4) can be removed / replaced. The plasma cell is presented in red. The numbers written above the components convey their middle position in meter as calculated from the cathode. Fig. 4(a), shows the current situation. Two possible scenarios for plasma cell arrangement in the PITZ beamline are shown in Figs. 4(b) and (c). In order to decide which scenario is preferable both possibilities were simulated. The calcu- 


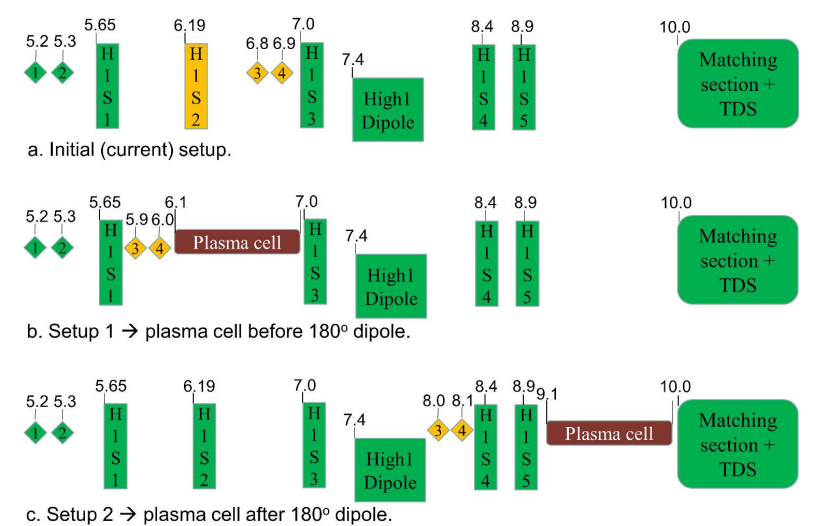

Figure 4: Block diagram of the relevant section of the PITZ beam line between ${ }^{222}$ booster and matching section/TDS. Frame (a) shows the current setup, frames ${ }^{223}$ (b) and (c) depict two possible scenarios for the plasma cell insertion.

lated electron beam parameters are very similar for both setups ${ }^{226}$ with the main difference being that the transverse beam focus- ${ }^{227}$ ing is easier achievable for Setup 1. This is illustrated in Fig. ${ }^{228}$ 5 , where the transverse electron beam sizes along the beam line ${ }^{229}$ for the two different setups are shown. The beam sizes at the ${ }^{230}$ plasma cell are very similar but the focusing gradient is lower ${ }^{231}$ for Setup 1 which relaxes alignment and makes it easier to cap- ${ }^{232}$ ture the beam at the exit of the plasma cell.

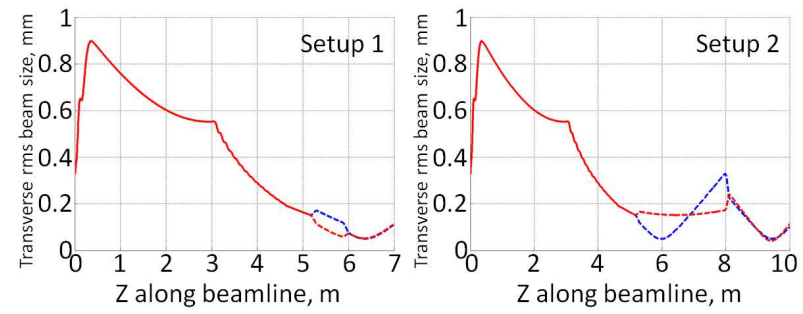

Figure 5: Transverse beam focusing for two different positions of the plasma ${ }^{244}$ cell. Different colors correspond to $\mathrm{rms}$ beam sizes in different transverse ${ }^{245}$ planes.

This means that a candidate position for the plasma cell insertion into the PITZ beam line was identified.

\section{Ionization of plasma channel}

The next step in preparing the self-modulation experiment 252 was to develop a concept for a plasma cell, generating and con-253 fining the plasma. There are three basic approaches: in plasma 254 discharge a high voltage pulse is applied to a gas cell, leading to 255 collision ionization of the gas. This is often done in capillaries256 and the main difficulty here is to generate a homogenous plasma257 density. The second approach is to heat a gas with an RF wave 258 (helicon wave). This works well for lower densities, but was 259 not shown yet for the densities (around $10^{15} \mathrm{~cm}^{-3}$ ) which are 260 targeted in the PITZ experiment. Therefore it was decided to 261 use laser ionization in a heat pipe oven, following the basic de-262 sign of Muggli et al. [16]. In this design Lithium or a different ${ }_{263}$ alkali metal with a low ionization potential is evaporated in a central pipe which also works as the beam pipe for the electron beam. The beginning and end of the Lithium zone is defined with a steep temperature gradient and Helium buffer gas. Thin windows separate the Helium zones at the outer ends from the beam pipe vacuum. Once the pressure regions have stabilized the Lithium gas is ionized with a laser which can be done in two ways:

1) Field ionization: this is a nonlinear process where a high intensity laser beam is used to ionize the metal vapor. This process is independent of the laser wavelength, important here is the strength of the electrical field of the laser wave. Above a certain threshold this field is strong enough to deform the atomic orbitals so far that the outer shell electrons are freed to ionize the atoms in a process called barrier suppressed ionization (BSI). An advantage of this process is that within the volume where the laser intensity is above that threshold complete ionization can be reached.

2) Single photon ionization: this is a linear process where light with a sufficiently short wavelength is absorbed by the metal vapor. The photon energy is high enough to transfer enough energy to the electrons to ionize them. UV light is needed for this process, e. g. with a wavelength of less than $231 \mathrm{~nm}$ in the case of Lithium, which has an ionization potential of $5.39 \mathrm{eV}$. This leads to partial ionization of the gas volume dependent on the laser intensity, asymptotically reaching full ionization for very high intensities.

Typically the ionization laser is coupled into the plasma cell on-axis [16]. This is not possible at PITZ in the above described scenarios because of space restrictions. Therefore the existing design concept of the heat pipe oven was modified to a cross shaped structure where the electron beam travels through one pipe and the ionizing laser is coupled into the oven through the orthogonal pipe. The four buffer gas regions are connected like the two regions in the original design and the orthogonal pipe is terminated with optical windows as coupling ports for the laser. An advantage of this configuration is the availability of side ports for optical characterization methods, e.g. interferometry for density measurement. Concepts based on the two ionization processes are described in the following.

\subsection{Field ionization}

For this scenario it is assumed to use a short pulse Ti:Sapphire laser to ionize Lithium vapor in the plasma cell. In order to assure complete ionization the BSI threshold intensity has to be reached which is $I_{t h}=3.4 \times 10^{12} \mathrm{~W} / \mathrm{cm}^{2}$. Since in our case we operate with short pulses of around $100 \mathrm{fs}$, the cw threshold value was adjusted following the calculations in [17] to $5 \times 10^{12} \mathrm{~W} / \mathrm{cm}^{2}$, which was used as the target intensity in the following simulations. According to experiences from the PIC simulations of the self-modulation experiment the minimum dimensions of the plasma channel was defined as $1 \mathrm{~mm}$ diameter and $60 \mathrm{~mm}$ length relative to the electron beam axis. A schematic of this ionization principle is shown in Fig. 6.

A view from the top is shown in Fig. 6(a). The laser is coupled into the plasma chamber in $\mathrm{x}$-direction, perpendicular to the electron beam. Multiple passes of the laser are used 


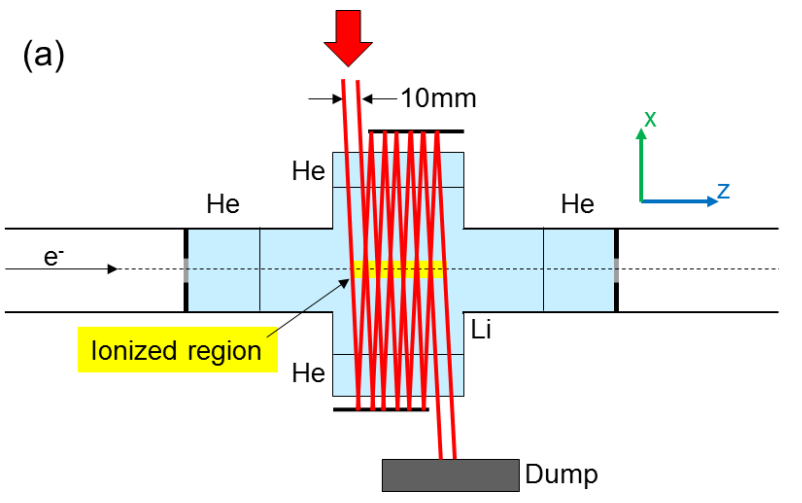

(b)

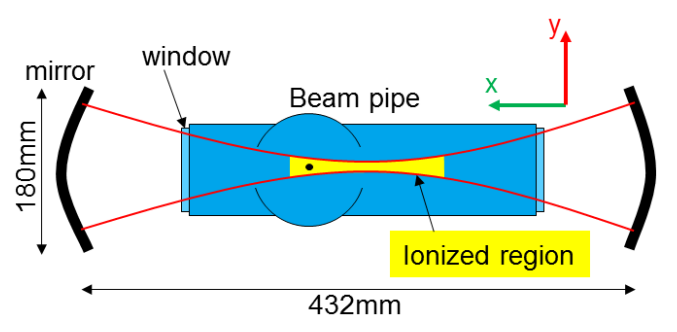

Figure 6: Scenario for plasma ionization with a Ti:Sapphire laser. Frame (a) is a view from top and frame (b) a view along the electron beam axis. Both sketches are not to scale. crossing of the laser beam with the electron beam axis.

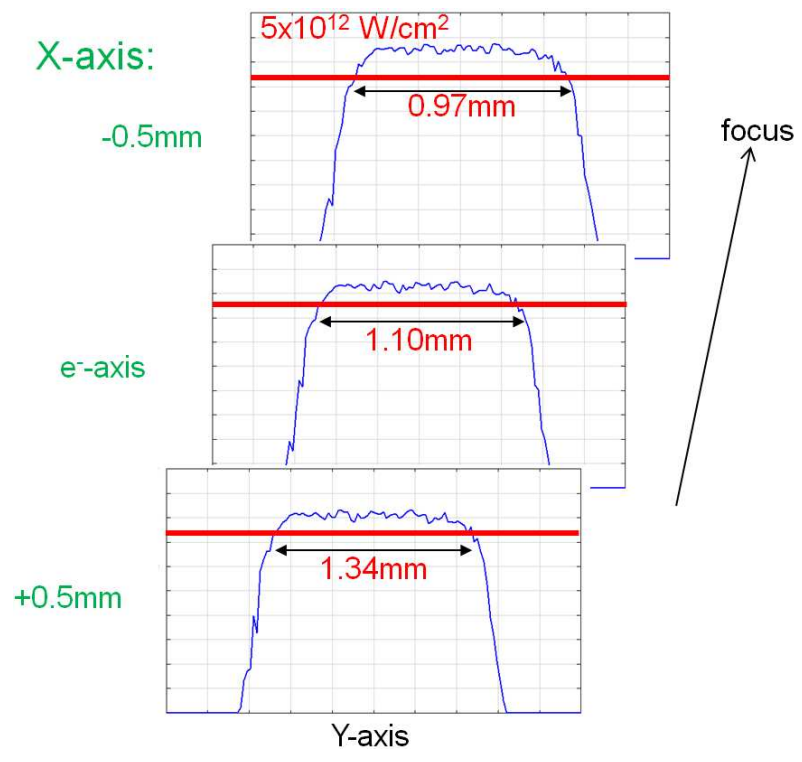

Figure 7: Simulation results of the optical intensity in a cut parallel to the vertical axis (y-axis).

The optical intensity distribution is shown for three positions along the $\mathrm{x}$-axis (propagation direction of the laser) around the electron beam axis. The horizontal bar (red) in each graph represents the BSI threshold intensity of $5 \times 10^{12} \mathrm{~W} / \mathrm{cm}^{2}$, indicating full ionization. The thickness of the ionized region in vertical direction (y-axis) is decreasing towards negative $\mathrm{x}$-values indication the approach to the focus. But the simulations show that a region with $1 \mathrm{~mm}$ diameter can be fully ionized perpendicular to the electron beam axis. Simulation results of the optical intensity in a cut parallel to the electron beam axis are shown in Fig. 8.

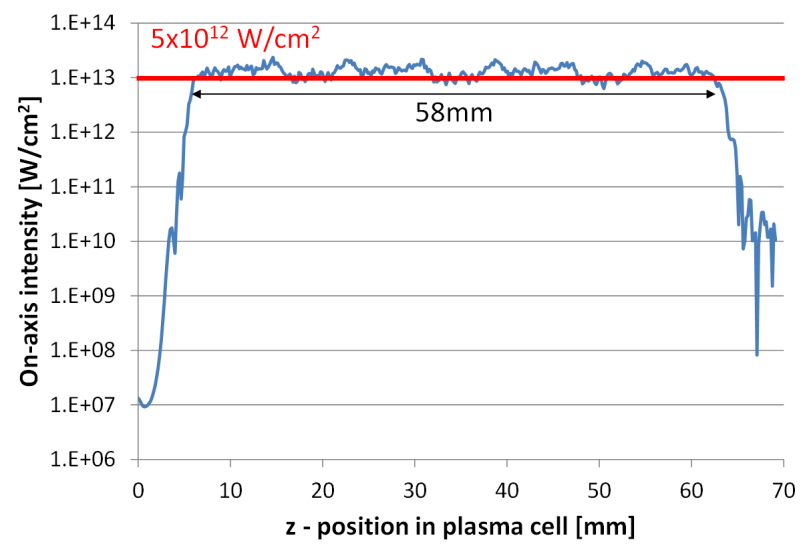

Figure 8: Simulation results of the optical intensity in a cut parallel to the electron beam axis.

As in Fig. 7, the horizontal bar (red) in Fig. 8 represents the BSI threshold intensity of $5 \times 10^{12} \mathrm{~W} / \mathrm{cm}^{2}$, indicating full ionization, in this case over a length of $58 \mathrm{~mm}$ along the electron beam axis. Together these simulations show that a plasma 
region can be generated using a $1 T W$ peak power Ti:Sapphire laser which is big enough for self-modulation experiments at PITZ

\subsection{Single photon ionization}

For this scenario it is assumed to use an $\mathrm{ArF}$ excimer laser to ionize Lithium vapor in the plasma cell. The ionization here is a linear process and the absorption of the laser energy $E$ by the neutral vapor is modeled by the Lambert-Beer law:

$$
E=E_{0} e^{-\alpha l}
$$

with the extinction coefficient $\alpha=\sigma_{x} N_{v}, \sigma_{x}$ representing the atomic cross-section and $N_{v}$ the number of atoms of the vapor per cubic unit. The principle setup is shown in Fig. 9.

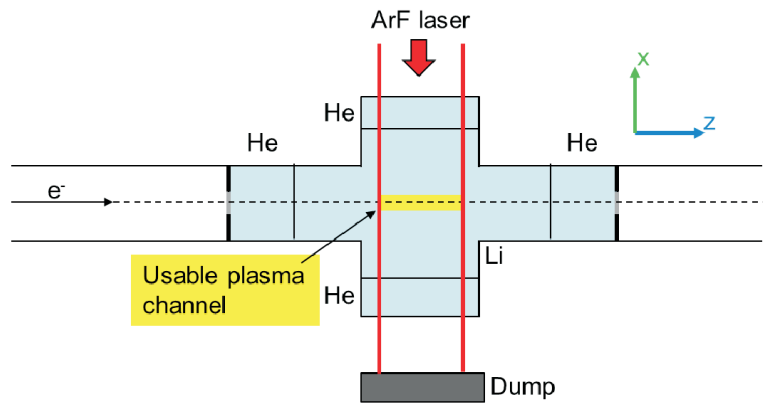

Figure 9: Scenario for plasma ionization with an ArF laser (View from top). ${ }^{352}$ Sketch is not to scale.

In the simplest scenario the ArF laser is passing just once ${ }_{355}$ through the vapor resulting in a certain plasma density in the $\mathrm{e}_{356}$ vicinity of the electron beam (,usable plasma channel“). But ${ }_{357}$ to utilize the laser energy more efficiently we modeled multi- ${ }_{358}$ pass configurations where the laser is reflected back into the ${ }_{359}$ plasma cell several times. The evolution of the plasma density ${ }_{360}$ was calculated by splitting the vapor column into normalized ${ }_{361}$ voxels, and recording the energy difference, $\Delta E$ from the beam ${ }_{362}$ entering and exiting each voxel for one pass through the sys- ${ }_{363}$ tem. Assuming the absorbed energy is quantized into photons $N_{p}=(\Delta E \cdot \lambda) /(h \cdot c)$ with the wavelength $\lambda$ and Planck's constant $h$ - and every photon absorbed equates to an ionization event re-364 leasing one electron. Thus we calculated the electron density, $N_{e}$ for each voxel and the corresponding reduction of the vapor 365 density $N_{v, n}=N_{v}-N_{e}$. For any further $\mathrm{n}^{\text {th }}$-pass the energy exit-366 ing the system is reinserted once again, with any potentially ap-367 plicable losses, and computed using the Beer-Lambert law on ${ }_{368}$ a voxel-by-voxel basis with the modified vapor density terms 369 for each voxel. The energy entering each voxel is equal to the 370 energy exiting the previous voxel. One can then summate the ${ }_{371}$ energy absorbed per voxel and directly relate that to the plasma 372 density in each voxel for however many reflections through the ${ }_{373}$ system. Fig. 10 shows achievable plasma densities as a func-374 tion of the vapor density and the number of passes.

The plasma density in the center of the plasma cell shows a 376 maximum which is a function of the vapor density and the ion-377 ization laser intensity. Reducing the vapor density results in $\mathrm{a}_{378}$

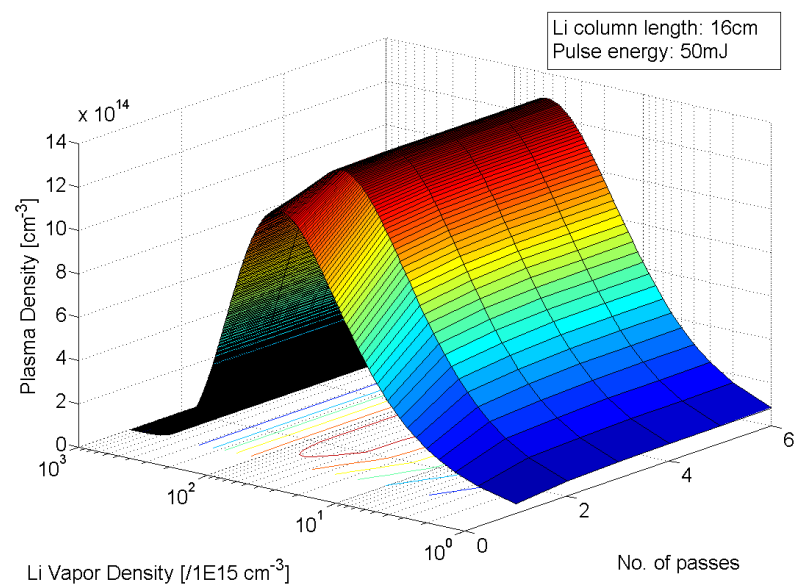

Figure 10: Calculated plasma density in the center of the plasma cell vs. vapor density and number of ionization passes for a given ionization laser intensity.

reduced plasma density since fewer atoms are available for ionization. Increasing the vapor density above the maximum leads to a reduced plasma density because less laser energy is reaching the center of the plasma cell, being absorbed beforehand. The plasma density for a particular voxel increases with each pass of the laser but most of the ionization is achieved with the first few passes owing to the strong reduction of the laser pulse energy due to absorption. Therefore the ionization setup should be implemented with a single pass or few passes at most to reach a plasma density of $10 \%$ of the vapor density. As an example, given a Lithium vapor density of $5 \times 10^{16}$ atoms $/ \mathrm{cm}^{3}$, which is the optimal value for the plasma cell setup given in Fig. 10, and 6 passes of the laser beam, a plasma density of $1.3 \times 10^{15} \mathrm{~cm}^{-3}$ is achieved. To generate a plasma channel of $10 \mathrm{~mm}$ diameter (for ease of electron beam alignment) and $60 \mathrm{~mm}$ length with a plasma density of $10^{15} \mathrm{~cm}^{-3}$ an ionization laser pulse energy of a few $100 \mathrm{~mJ}$ is needed. An ArF excimer laser with $400 \mathrm{~mJ}$ pulse energy was bought by PITZ together with the University of Hamburg for the self-modulation experiment.

\section{Design of plasma cell}

Based on the results as presented in the last section it was decided to design and build a cross-shaped plasma cell for the PITZ experiment which is shown as a design model in Fig. 11.

The length of the plasma cell in electron beam direction (beam pipe) of $500 \mathrm{~mm}$ is restricted by the available space in the PITZ beam line but is sufficient to generate a homogeneous Lithium vapor column of $100 \mathrm{~mm}$ length [18]. Following the plasma cell design in [16] the Lithium column in the beam pipe is buffered on both sides with Helium which is cooled with water cooling jackets. The beam pipe is terminated on both sides with $25 \mu \mathrm{m}$ thick Kapton foils which separate the Lithium/Helium atmosphere within the plasma cell from the beam vacuum when inserted into the PITZ beam line. The inside of the oven will be covered with a steel mesh functioning as 


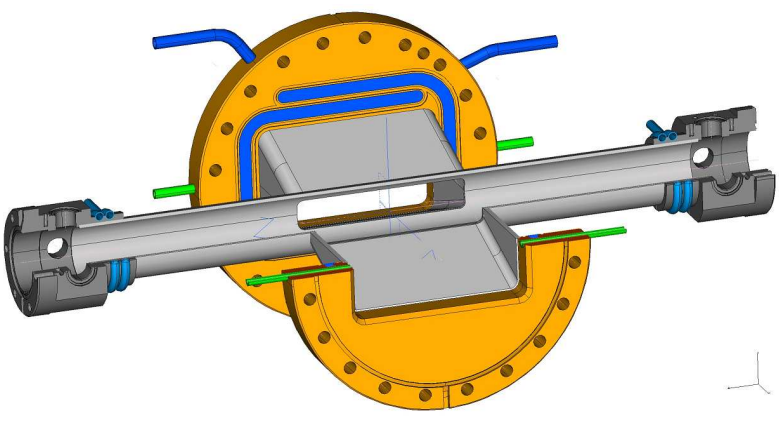

Figure 11: Design model of the PITZ plasma cell.The beam pipe is going from ${ }^{426}$ right to left, terminated on both sides with cooling jackets. The side openings 427 will be used to couple the ionization laser into the plasma cell. More details are 428 in the text.

a wick of the heat pipe oven. The orthogonal pipe was designed as a feed of the ionization laser, especially taking into account the dimensions needed for the Ti:Sapphire laser. The opening ${ }^{431}$ of the beam pipe to the sides is $20 \mathrm{~mm} \times 120 \mathrm{~mm}$ to allow side $\mathrm{e}^{432}$ ionization of the plasma channel. From there the side parts are ${ }_{434}$ opening up to allow expansion of the laser beam towards the 435 outer mirrors. When using an ArF laser this cone shaped ex-436 pansion could be utilized for multipass ionization. The ends of ${ }_{438}^{437}$ the orthogonal pipe are buffered with Helium in the same way ${ }_{439}$ as the beam pipe to prevent that Lithium covers the laser en-440 trance windows which are terminating the sides. Water cooling ${ }^{441}$ is used here in the same way as in the cooling jackets of the ${ }_{443}^{42}$ beam pipe. All four Helium areas are connected for pressure ${ }_{444}$ equalization.

\section{Summary}

Self-modulation of particle beams in a plasma was proposed ${ }_{455}^{450}$ as a new concept to enable plasma wakefield acceleration with ${ }^{452}$ long driver beams. Since this concept was not demonstrated ${ }^{453}$ unambiguously yet experimentally, an experiment is in prepa ${ }_{455}^{454}$ ration at PITZ (DESY, Zeuthen site) to demonstrate and char- ${ }^{-456}$ acterize self-modulation of an electron beam. Key elements for ${ }^{457}$ this are the highly flexible photocathode laser system and the ${ }^{458}$ well-developed beam diagnostics. To show the feasibility of ${ }_{460}^{459}$ this proposal, PIC simulations based on PITZ electron beam ${ }_{461}$ and expected plasma parameters were conducted, showing a $^{462}$ well formed self-modulation signal in the longitudinal phase ${ }_{464}^{463}$ space.

Preparations for the experiment have started at PITZ. In a 466 first step a suitable insertion point for the plasma cell with the ${ }^{467}$ middle of the plasma cell about $6.4 \mathrm{~m}$ downstream the photo- ${ }_{469}^{468}$ cathode was determined with beam dynamics simulations. The ${ }_{470}^{469}$ available space for the plasma cell plus periphery is about $1 m_{471}$ which is sufficient. It was decided to use laser ionization to generate the plasma since this technique is capable of providing a homogeneous plasma channel with sufficient size ( $1 \mathrm{~mm}$ diameter, $60 \mathrm{~mm}$ length) and density $\left(10^{15} \mathrm{~cm}^{-3}\right)$. Two different ways were found to generate the plasma, utilizing either field ionization with a $1 T W$ Ti:Sapphire laser or single photon ionization with a $400 \mathrm{~mJ} \mathrm{ArF}$ excimer laser. As opposed to previously realized designs the ionization laser is coupled from the side, orthogonally to the electron beam direction. A plasma cell fitting all the needs was designed and is currently under construction.

\section{Acknowledgements}

Patric Muggli is acknowledged for his tremendous help in all aspects of the design of the experiment. We are also very thankful for fruitful discussions with members of the AWAKE and LAOLA collaborations. Instrumental was also the support within PITZ, especially the help of Alexander Donat, Gerald Koss and Dieter Richter ${ }^{1}$. The work was partially supported by the German federal ministry of education and research, project 05H12GU6.

\section{References}

[1] T. Tajima, J. M. Dawson, „Laser electron accelerator“, Phys. Rev. Lett. 43, 267-270 (1979)

[2] I. Blumenfeld et al., ,Energy doubling of $42 \mathrm{GeV}$ Electrons in a meterscale plasma wakefield accelerator", Nature 445, 741-744 (2007)

[3] A. Caldwell et al., „Proton-driven plasma-wakefield acceleration“, Nature Physics 5, 363-367 (2009)

[4] N. Kumar, A. Pukhov, and K. Lotov, ,Self-Modulation Instability of a Long Proton Bunch in Plasmas“, Phys. Rev. Lett. 104, 255003 (2010)

[5] C. Schroeder et al., ,Growth and Phase Velocity of Self-Modulated BeamDriven PlasmaWaves“, Phys. Rev. Lett. 107, 145002 (2011)

[6] J. Vieira et al., „Transverse self-modulation of ultra-relativistic lepton beams in the plasma wakefield accelerator", Phys. Plasmas 19, 063105 (2012)

[7] Y. Fang et al, submitted

[8] A. Martinez de la Ossa et al. „Self-modulation of long electron beams in plasma at PITZ“, AIP Conf. Proc. 1507, 588-593 (2012)

[9] F. Stephan et al. „Detailed characterization of electron sources yielding first demonstration of European X-ray Free-Electron Laser beam quality“, Phys. Rev. STAB 13, 020704 (2010)

[10] D. Malyutin et al. „Commissioning of new Diagnostics Devices at PITZ“, Proceedings of the XXIII Russian Particle Accelerator Conference, Peterhof, St. Petersburg, Russia, 2012, WEPPD057

[11] I. Will et al. „Photoinjector drive laser of the FLASH FEL“, Opt. Exp. 19, 23770 (2011)

[12] I. Will, G. Klemz, „Generation of flat-top picosecond pulses by coherent pulse stacking in a multicrystal birefringent filter", Opt. Exp. 16, 14922 (2008)

[13] R. Fonseca, L. Silva, F. Tsung, V. Decyk, W. Lu, C. Ren, W. B. Mori, S. Deng, S. Lee, T. Katsouleas, and J. Adam, Notes Comp. Sci. 2331, 342-351 (2002)

[14] Malyutin et al. „Simulation of the Longitudinal Phase Space Measurements With the Transverse Deflecting Structure at PITZ“, Proc. of IPAC 2012, MOPPP034

[15] K. Flöttmann, ASTRA, http://www.desy.de/ mpyflo

[16] P. Muggli et al. „Photo-Ionized Lithium Source for Plasma Accelerator Applications“, IEEE Trans Plasma Science 27, 791 (1999)

[17] X. Tong, C. Lin, „Empirical formula for static field ionization rates of atoms and molecules by lasers in the barrier-suppression regime“, J. Phys. B: At. Mol. Opt. Phys. 38, 2593 (2005)

[18] P. Muggli et al., „Energy scaling with plasma length and density in the plasma wakefield accelerator", New J. Phys. 12, 045022 (2010)

${ }^{1}$ Helmholtz-Zentrum Berlin für Materialien und Energie Berlin 